\title{
Improved Receiver Design for Layered ACO-OFDM in Optical Wireless Communications
}

\author{
Qi Wang, Zhaocheng Wang, Senior Member, IEEE, Xuhan Guo, and Linglong Dai, Senior Member, IEEE
}

\begin{abstract}
Layered asymmetrically clipped optical orthogonal frequency division multiplexing (LACO-OFDM) is recently proposed for intensity-modulated directed-detected optical wireless communications, which achieves higher spectral efficiency compared with the conventional ACO-OFDM, since different layers of ACO-OFDM signals are combined to utilize more subcarriers. In this letter, an improved receiver is proposed for LACO-OFDM, which distinguishes different layers of ACO-OFDM signals in the time domain. After that, the structure of ACO-OFDM signals in each layer is exploited to further reduce the noise and inter-layer interference, resulting in the improved performance. Simulation results show that the proposed receiver for LACO-OFDM achieves significant gain over its conventional counterpart.
\end{abstract}

Index Terms-Layered asymmetrically clipped optical orthogonal frequency division multiplexing (LACO-OFDM), intensity modulation with direct detection (IM/DD), optical wireless communication (OWC), iterative receiver.

\section{INTRODUCTION}

D UE TO the rapid development of solid-state lighting technologies, optical wireless communication (OWC) has attracted increasing attention from both academia and industry in recent years. With its distinct features such as broad unregulated spectrum, low power consumption and ubiquitous connection, OWC has been regarded as a promising technique for indoor wireless networks [1], [2].

As a spectrally efficient technique, orthogonal frequency division multiplexing (OFDM) has been utilized in OWC systems because of its inherent resistance to inter symbol interference (ISI) and simple one-tap equalization at the receiver [3], [4]. Since OWC systems usually employ intensity modulation with direct detection (IM/DD) for low cost implementation, the transmitted signal has to be realvalued and non-negative, and conventional OFDM needs to

Manuscript received September 27, 2015; revised October 16, 2015; accepted October 24, 2015. Date of publication October 27, 2015; date of current version December 22, 2015. This work was supported in part by the National Key Basic Research Program of China under Grant 2013CB329203, in part by the Shenzhen Peacock Plan under Grant 1108170036003286, in part by the Shenzhen Wireless over Visible Light Communication Technology Engineering Laboratory under Grant SDRC[2012] 1440, in part by the Shenzhen Visible Light Communication System Key Laboratory under Grant ZDSYS20140512114229398, and in part by the National Natural Science Foundation of China under Grant 61271266.

Q. Wang, Z. Wang, and L. Dai are with the Tsinghua National Laboratory for Information Science and Technology, Department of Electronic Engineering, Tsinghua University, Beijing 100084, China (e-mail: qiwang11@mails.tsinghua.edu.cn; zcwang@tsinghua.edu.cn; daill@tsinghua. edu.cn).

$\mathrm{X}$. Guo is with the Electrical Engineering Division, Centre for Photonic Systems, Department of Engineering, University of Cambridge, Cambridge CB3 0FA, U.K. (e-mail: xg218@cam.ac.uk).

Color versions of one or more of the figures in this letter are available online at http://ieeexplore.ieee.org.

Digital Object Identifier 10.1109/LPT.2015.2495320 be modified. Hermitian symmetry is commonly applied to the OFDM subcarriers to obtain real time-domain signals [5]-[7]. Furthermore, several modified optical OFDM schemes have been proposed to make the signals non-negative, namely, DC-biased optical OFDM (DCO-OFDM), asymmetrically clipped optical OFDM (ACO-OFDM), pulse-amplitudemodulated discrete multitone (PAM-DMT), and unipolar OFDM (U-OFDM) [8]-[11]. In DCO-OFDM, a DC bias is added to the bipolar signals, resulting in the increased transmitted power. ACO-OFDM is proposed in [8], which only modulates the odd subcarriers and generates antisymmetric signals in the time domain. The time-domain signals can be directly clipped at zero and the clipping distortion only falls on the even subcarriers. In PAM-DMT, only the imaginary part of each subcarrier is modulated and the clipping distortion falls on the real part of the same subcarrier [9]. U-OFDM or Flip OFDM is proposed in [10] and [11], where the positive and inverted negative signals are transmitted in two different time slots. Although ACO-OFDM, PAM-DMT and U-OFDM do not need DC bias and achieve high power efficiency, the spectral efficiency is sacrificed by half. Layered ACO-OFDM (LACO-OFDM) is recently proposed in [12], which combines different layers of ACO-OFDM signals occupying different subcarriers for simultaneous transmission. Almost all the subcarriers can be used for modulation in LACO-OFDM, which improves the spectral efficiency significantly compared with conventional ACO-OFDM.

In conventional receiver for LACO-OFDM, the symbols on the lowest layer are firstly detected by fast Fourier transform (FFT). Afterwards, the symbols on higher layers are recovered by subtracting the estimated clipping distortion of the lower layers [12]. However, this frequencydomain detection method cannot fully exploit the structure of LACO-OFDM signals, which limits its performance. In this letter, an improved receiver is proposed for LACO-OFDM, which distinguishes different layers of ACO-OFDM signals in the time domain. After that, the structure of ACO-OFDM signals in each layer is exploited to further reduce the noise and inter-layer interference, resulting in improved performance. Simulation results illustrate that the proposed receiver for LACO-OFDM outperforms its conventional counterpart.

\section{LAYERED ACO-OFDM FOR OWC SYSTEMS}

In LACO-OFDM with $N$ subcarriers and $L$ layers, the transmitted bit stream is mapped onto quadrature amplitude modulation (QAM) symbols $X_{k}, k=0,1, \cdots, N-1$, which are divided into $L$ groups for layered modulation. In Layer $l(l=1,2, \cdots, L)$ ACO-OFDM, we consider an OFDM symbol in which only the $2^{l-1} k$-th subcarriers are modulated, denoted as $X_{\mathrm{ACO}, k}^{(l)}, k=0,1, \cdots, N / 2^{l-1}-1$. 
After N-point inverse FFT (IFFT), the time-domain signals $x_{\mathrm{ACO}, n}^{(l)}, n=0,1, \ldots, N-1$ can be obtained. Only the subcarriers with odd indexes of $X_{\mathrm{ACO}, k}^{(l)}$ are used to carry useful information, while the subcarriers with even indexes of $X_{\mathrm{ACO}, k}^{(l)}$ are set to zero as in conventional ACO-OFDM [8]. Therefore, $N / 2^{l+1}$ useful symbols are actually assigned to Layer $l$ ACO-OFDM, and different layers occupy orthogonal subcarriers.

The time-domain signals have the antisymmetric characteristic that $x_{n}^{(l)}=-x_{n+N / 2^{l}}^{(l)}$, which can be directly clipped at zero without information loss [12]. The clipped Layer $l$ ACO-OFDM signals are denoted as

$\left\lfloor x_{\mathrm{ACO}, n}^{(l)}\right\rfloor_{c}=x_{\mathrm{ACO}, n}^{(l)}+i_{\mathrm{ACO}, n}^{(l)}= \begin{cases}x_{\mathrm{ACO}, n}^{(l)}, & x_{\mathrm{ACO}, n}^{(l)} \geq 0 \\ 0, & x_{\mathrm{ACO}, n}^{(l)}<0\end{cases}$

for $n=0,1, \ldots, N-1$, where $i_{\mathrm{ACO}, n}^{(l)}$ denotes the negative clipping distortion of Layer $l$ ACO-OFDM.

In LACO-OFDM scheme, different layers of ACO-OFDM are combined in the time domain for simultaneous transmission. The time-domain LACO-OFDM signals with $L$ layers are written as $x_{\mathrm{L}-\mathrm{ACO}, n}=\sum_{l=1}^{L}\left\lfloor x_{\mathrm{ACO}, n}^{(l)}\right\rfloor_{c}, n=0,1, \ldots, N-1$.

\section{RECEIVER DESIGN}

At the receiver, the optical signal is converted to the electric signal by avalanche photodiode (APD). After analog to digital $(\mathrm{A} / \mathrm{D})$ conversion and $\mathrm{CP}$ removal, the digital signal is used to recover the transmitted information. Since the shot noise and thermal noise is usually modeled as additive white Gaussian noise (AWGN) [13], [14], and the received signal is given by $r_{n}=x_{\mathrm{L}-\mathrm{ACO}, n}+w_{n}, n=0,1, \ldots, N-1$, where $w_{n}$ denotes the samples of AWGN with zero mean and variance of $\sigma^{2}$. It can be rewritten in the frequency-domain form after FFT as $R_{k}=X_{\mathrm{L}-\mathrm{ACO}, k}+W_{k}$ for $k=0,1, \ldots, N-1$.

\section{A. Conventional Receiver}

In [12], a frequency-domain receiver is proposed for LACO-OFDM. Since both the useful symbols and clipping distortion in layer $2 \sim L$ ACO-OFDM are on the even subcarriers, the symbols on Layer 1 ACO-OFDM can be directly detected after FFT of $r_{n}$ as [12]

$\hat{X}_{\mathrm{ACO}, k}^{(1)}=\arg \min _{X \in \mathcal{S}}\left|X-2 \hat{R}_{\mathrm{ACO}, k}^{(1)}\right|, \quad k=1,3, \ldots, N / 2-1$,

where $\mathcal{S}$ denotes the constellation set for modulation.

For Layer $l(l>1)$ ACO-OFDM, the negative clipping distortion from Layer $1 \sim l-1$ ACO-OFDM will interfere the useful symbols, which should be removed before detection. The time-domain signals of Layer $m(m<l)$ ACO-OFDM $\left\lfloor\hat{x}_{\mathrm{ACO}, n}^{(m)}\right\rfloor_{c}$ can be reconstructed by $\hat{X}_{\mathrm{ACO}, k}^{(m)}$. Afterwards, the frequency-domain clipping distortion for Layer $m$ ACO-OFDM can be estimated by FFT of $\left\lfloor\hat{x}_{\mathrm{ACO}, n}^{(m)}\right\rfloor_{c}$, which is denoted as $\hat{I}_{\mathrm{ACO}, 2^{l-m} k}^{(m)}$ and should be subtracted from the frequency-domain symbols as [12]

$$
\hat{R}_{\mathrm{ACO}, k}^{(l)}=R_{2^{l-1} k}-\sum_{m=1}^{l-1} \hat{I}_{\mathrm{ACO}, 2^{l-m} k}^{(m)}
$$

for $k=0,1, \ldots, N / 2^{l-1}-1$. Similar to (2), the transmitted symbols in Layer $l$ ACO-OFDM can be detected by

$$
\hat{X}_{\mathrm{ACO}, k}^{(l)}=\arg \min _{X \in \mathcal{S}}\left|X-2 \hat{R}_{\mathrm{ACO}, k}^{(l)}\right| .
$$

\section{B. Proposed Receiver}

Although the above conventional receiver is simple and straightforward, it does not fully exploit the structure of LACO-OFDM signals, which limits its performance. In the following, we propose a novel receiver to further improve the performance of LACO-OFDM, which distinguishes different layers of ACO-OFDM signals in the time domain and pairwise clipping is utilized in each layer.

In the proposed receiver, the signals in different layers of ACO-OFDM signals are firstly separated in the time domain. The symbols on Layer 1 ACO-OFDM is also detected after FFT as in (2). The time-domain signals in Layer 1 ACO-OFDM are reconstructed by

$\hat{r}_{n}^{(1)}=\left\lfloor\sum_{k=0}^{N-1} \hat{X}_{\mathrm{ACO}, k}^{(1)} \exp \left(j \frac{2 \pi}{N} n k\right)\right\rfloor_{c}, \quad n=0,1, \ldots, N-1$,

where $\hat{X}_{\mathrm{ACO}, k}^{(1)}=\left(\hat{X}_{\mathrm{ACO}, N-k}^{(1)}\right)^{*}$.

Afterwards, the reconstructed signals $\hat{r}_{n}^{(1)}$ are discarded from the original received signals $r_{n}$, where the remaining signals $\tilde{r}_{n}^{(2)}=r_{n}-\hat{r}_{n}^{(1)}$ can be considered as the combination of the signals in Layer $2 \sim l$ ACO-OFDM. Since the signals $\hat{r}_{n}^{(1)}$ containing both the transmitted symbols and clipping distortion in Layer 1 ACO-OFDM are already removed, the symbols in Layer 2 ACO-OFDM are be directly detected after FFT as well similar to (2).

When the symbols in Layer $1 \sim l-1(l>1)$ ACO-OFDM are detected, all of their reconstructed signals are discarded from the original received signals $r_{n}$, and we have

$$
\tilde{r}_{n}^{(l)}=r_{n}-\sum_{m=1}^{l-1} \hat{r}_{n}^{(m)}=\tilde{r}_{n}^{(l-1)}-\hat{r}_{n}^{(l-1)}
$$

where we denote $\tilde{r}_{n}^{(1)}=r_{n}$ and the reconstructed time-domain signals in Layer $l$ ACO-OFDM are given by

$$
\hat{r}_{n}^{(l)}=\left\lfloor\sum_{k=0}^{N-1} \hat{X}_{\mathrm{ACO}, k}^{(l)} \exp \left(j \frac{2 \pi}{N} n \cdot 2^{l-1} k\right)\right\rfloor_{c}
$$

for $n=0,1, \ldots, N-1$.

The symbols in Layer $l$ ACO-OFDM can be directly detected after the FFT of $\tilde{r}_{n}^{(l)}$. It can be seen that in LACO-OFDM with $L$ layers, $\tilde{r}_{n}^{(l)}$ contains more than one layers of ACO-OFDM when $l<L$. However, $\tilde{r}_{n}^{(L)}$ only includes the signals in Layer $L$ ACO-OFDM since all the signals from layer $1 \sim L-1$ have been removed. Therefore, the structure of Layer $L$ ACO-OFDM can be utilized to further improve the performance.

We denote the received signals with only Layer $l$ ACO-OFDM as $\bar{r}_{n}^{(l)}=\left\lfloor x_{n}^{(l)}\right\rfloor_{c}+w_{n}+e_{n}^{(l)}$, where $e_{n}^{(l)}$ is the inter-layer interference and it follows a Gaussian distribution according to the central limit theorem, and we have $\bar{r}_{n}^{(l)}=\tilde{r}_{n}^{(L)}$. 
In Layer $l$ ACO-OFDM, the transmitted time-domain signals are periodic [12]. Besides that, asymmetric clipping is imposed on the time-domain signals so that either $\left[x_{n}^{(l)}\right\rfloor_{c}$ or $\left\lfloor x_{n+N / 2^{l}}^{(l)}\right\rfloor_{c}$ is zero when they are transmitted, and the remaining signal is non-negative. Therefore, we could estimate which one is zero according to $\bar{r}_{n}^{(l)}$ and then set it to zero, and half of the noise and inter-layer interference are eliminated. Pairwise maximum likelihood detector has been proposed in [15] to eliminate the extra noise for conventional ACO-OFDM. In Layer $l$ ACO-OFDM, however, the periodicity of the signals should also be considered and the pairwise clipping is modified as

$$
\bar{r}_{n, c}^{(l)}= \begin{cases}\bar{r}_{n}^{(l)} I_{H\left(n^{\prime}\right)}, & n^{\prime} \leq N / 2^{l} \\ \bar{r}_{n}^{(l)}\left(1-I_{H\left(n^{\prime}-N / 2^{l}\right)}\right), & n^{\prime}>N / 2^{l}\end{cases}
$$

where $n^{\prime}=\bmod \left(n, N / 2^{l-1}\right)$ and $I_{\{A\}}$ is an indicator function with $I_{\{A\}}=1$ if the event $A$ is true and $I_{\{A\}}=0$ otherwise. $H\left(n^{\prime}\right)$ is defined as

$$
H\left(n^{\prime}\right): \sum_{m=0}^{2^{l-1}-1} \bar{r}_{n^{\prime}+m N / 2^{l-1}}^{(l)} \leq \sum_{m=0}^{2^{l-1}-1} \bar{r}_{n^{\prime}+N / 2^{l}+m N / 2^{l-1}}^{(l)},
$$

Pairwise clipping is firstly utilized in Layer $L$ ACO-OFDM signals since $\bar{r}_{n}^{(L)}$ can be obtained after all the other layers are removed. Afterwards, the pairwise clipped signal is used to demodulate the symbols in Layer $L$ ACO-OFDM, which can achieve better performance compared with conventional method since half of the noise and inter-layer interference have been eliminated. Afterwards, the received time-domain signals in Layer $L$ ACO-OFDM are reconstructed by (7).

Since the time-domain signals in each layer have been reconstructed according to (7), the received signals with only Layer $l$ ACO-OFDM can be obtained by subtracting the signals in the other layers from the received signals as

$$
\bar{r}_{n}^{(l)}=r_{n}-\sum_{m \neq l} \hat{r}_{n}^{(m)},
$$

so that different layers of ACO-OFDM signals are distinguished in the time domain and pairwise clipping can be applied to eliminate half of the noise and inter-layer interference.

The symbols on Layer $l$ ACO-OFDM are detected again after pairwise clipping and FFT, where more accurate results are obtained, which could be used to update the signals $\hat{r}_{n}^{(l)}$ and back substitute to (10) to update the signals in other layers. Therefore, the proposed receiver operates in an iterative way. In each iteration, the signals in each layer are sequentially detected, and the reconstructed time-domains signals are used to update the signals in other layers. The pseudocode of the proposed receiver is summarized in Algorithm 1, where iter denotes the number of iterations.

\section{NumericAl RESUlts}

The bit error rate (BER) performance of the proposed receiver is evaluated by simulations in terms of bit energy to noise power ratio $E_{b} / N_{0}$. The number of IFFT used at the transmitter is 512 . The subcarriers are modulated by 16QAM in all layers, and the power of each modulated subcarrier is the

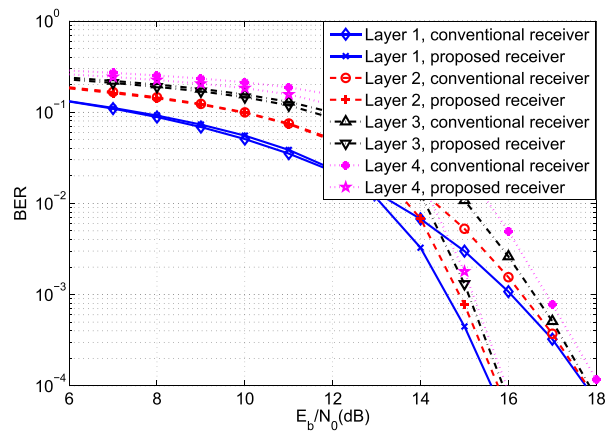

Fig. 1. BER performance comparison of the proposed receiver and conventional receiver for LACO-OFDM with 16QAM and 4 layers, where the BER of each layer of ACO-OFDM is calculated separately.

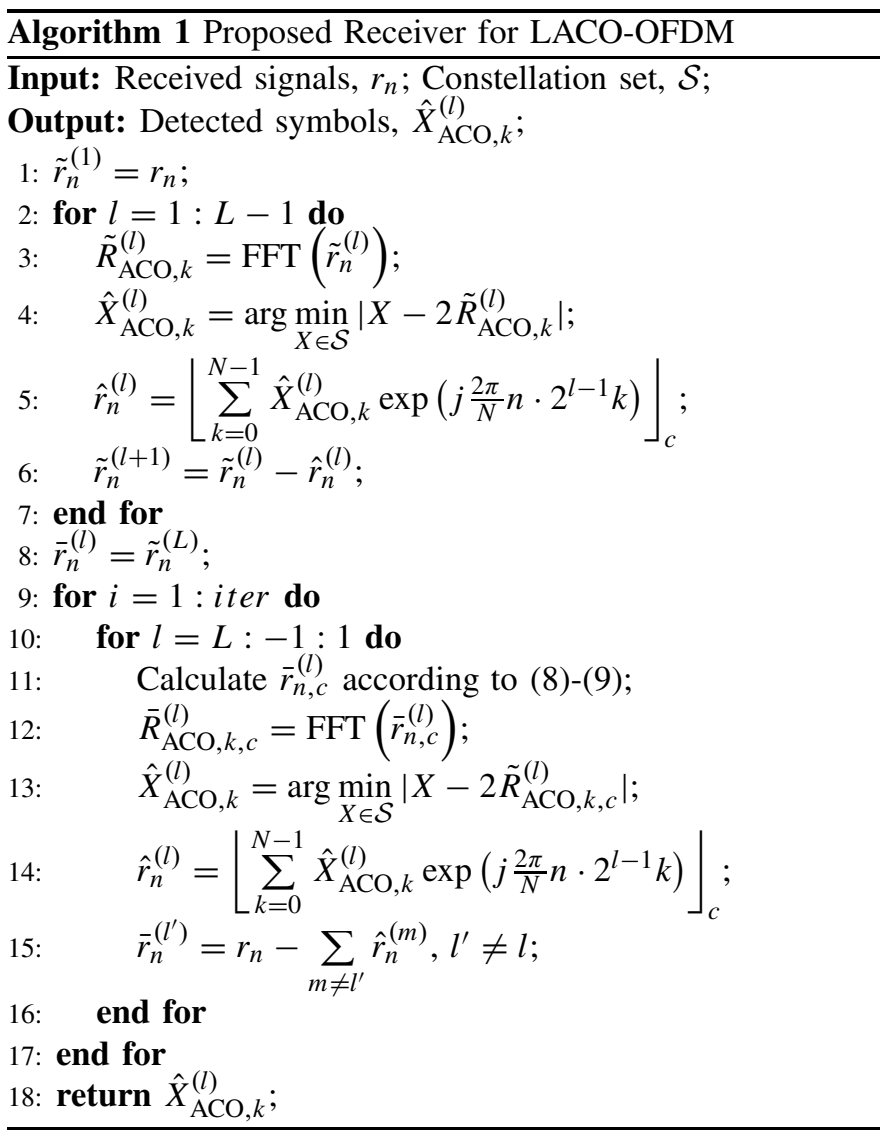

same in each layer. The number of iterations in the proposed receiver is set to 2 so that the complexity is relatively low.

Figure 1 shows the BER performance of LACO-OFDM with 4 layers, where the BER in each layer of ACO-OFDM is calculated separately. It can be seen that the proposed receiver achieves much better performance compared with the conventional receiver in all the four layers. More specifically, the performance gains for Layer 1-4 ACO-OFDM are $1.45-1.7 \mathrm{~dB}$ at BER of $10^{-3}$, and they are increased to $2-2.15 \mathrm{~dB}$ at BER of $10^{-4}$ since the estimation becomes more accurate when the BER is lower.

The performance of the proposed receiver is also validated by the average BER of LACO-OFDM with different layers, where BERs of all layers in LACO-OFDM are averaged and the simulation results are shown in Fig. 2. The numbers of 


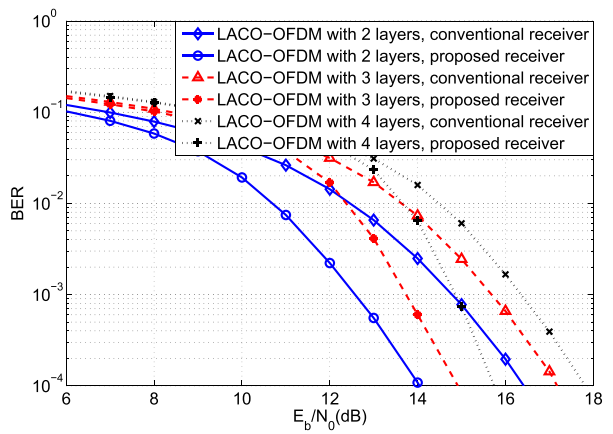

Fig. 2. BER performance comparison of the proposed receiver and conventional receiver for LACO-OFDM with different layers.

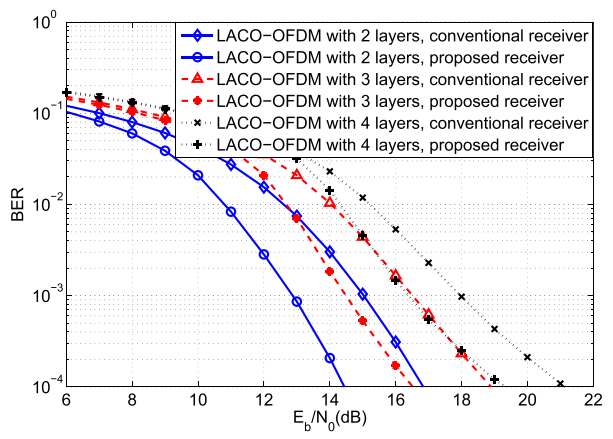

Fig. 3. BER performance comparison between the conventional receiver and the proposed one under the influence of the transmitter nonlinearity.

layers are set to 2, 3 and 4 , and the corresponding spectral efficiencies are $1.5 \mathrm{bit} / \mathrm{s} / \mathrm{Hz}, 1.75 \mathrm{bit} / \mathrm{s} / \mathrm{Hz}$ and $1.875 \mathrm{bit} / \mathrm{s} / \mathrm{Hz}$, respectively. It can be seen that for different kinds of LACO-OFDM, the proposed receiver always outperforms the conventional counterpart. LACO-OFDM with larger number of layers performs worse since higher spectral efficiency is achieved similar to [12]. At BER of $10^{-3}$, the performance gains are $2.2 \mathrm{~dB}, 2.15 \mathrm{~dB}, 1.5 \mathrm{~dB}$ for LACO-OFDM with 2,3 and 4 layers, respectively. When the BER reduces to $10^{-4}$, the performance gains are further increased to $2.4 \mathrm{~dB}, 2.25 \mathrm{~dB}$, $2.1 \mathrm{~dB}$, respectively.

The performance of the proposed receiver is also evaluated in Fig. 3 under the existence of transmitter nonlinearity. A simple double-clipped nonlinearity model is considered as (26) in [13], where the linear range is set to $[0,5]$ and the average power of each modulated symbol is normalized. It can be seen that the performance gains of the proposed receiver are still around $2 \mathrm{~dB}$ for all the three LACO-OFDM schemes.

Furthermore, the relationship between the BER performance and the number of iterations is investigated in Fig. 4, where LACO-OFDM with 4 layers is considered without transmitter nonlinearity. When larger number of iterations is used in the proposed receiver, better performance can be achieved. However, when the number of iterations is larger than 2, the performance improvement is limited with the increase of iterations, while higher complexity and latency are required. Therefore, there should be a tradeoff between complexity and performance for various applications.

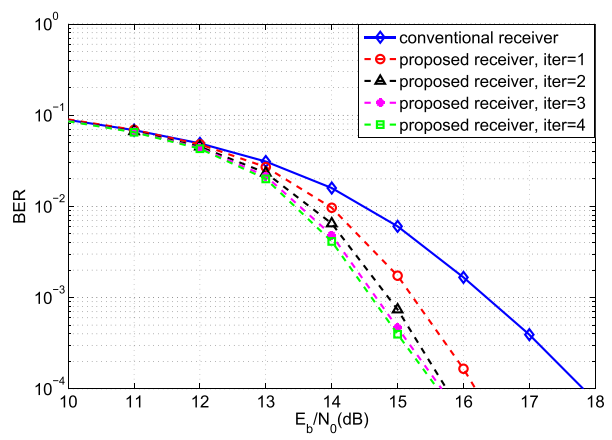

Fig. 4. BER performance comparison of the proposed receiver for LACOOFDM with different iterations, where 16QAM and 4 layers are utilized.

\section{CONCLUSIONS}

In this letter, an improved receiver is proposed for LACO-OFDM in OWC systems, which takes advantage of the structure of LACO-OFDM to further reduce the noise and inter-layer interference, resulting in the improved performance. The different layers of ACO-OFDM signals in LACO-OFDM are distinguished in the time domain and detected in an iterative way. Simulation results demonstrate that the proposed receiver for LACO-OFDM achieves significant gain over its conventional counterpart.

\section{REFERENCES}

[1] L. Hanzo, H. Haas, S. Imre, D. O’Brien, M. Rupp, and L. Gyongyosi, "Wireless myths, realities, and futures: From $3 \mathrm{G} / 4 \mathrm{G}$ to optical and quantum wireless," Proc. IEEE, vol. 100, no. Special Centennial Issue, pp. 1853-1888, May 2012.

[2] R. Zhang, J. Wang, Z. Wang, Z. Xu, C. Zhao, and L. Hanzo, "Visible light communications in heterogeneous networks: Paving the way for user-centric design," IEEE Wireless Commun., vol. 22, no. 2, pp. 8-16, Apr. 2015.

[3] D. Tsonev et al., "A 3-Gb/s single-LED OFDM-based wireless VLC link using a Gallium nitride $\mu$ LED," IEEE Photon. Technol. Lett., vol. 26, no. 7, pp. 637-640, Apr. 1, 2014.

[4] Q. Wang, Z. Wang, and L. Dai, "Asymmetrical hybrid optical OFDM for visible light communications with dimming control," IEEE Photon. Technol. Lett., vol. 27, no. 9, pp. 974-977, May 1, 2015.

[5] J. Armstrong, "OFDM for optical communications," J. Lightw. Technol., vol. 27, no. 3, pp. 189-204, Feb. 1, 2009.

[6] X. Li, J. Vucic, V. Jungnickel, and J. Armstrong, "On the capacity of intensity-modulated direct-detection systems and the information rate of ACO-OFDM for indoor optical wireless applications," IEEE Trans. Commun., vol. 60, no. 3, pp. 799-809, Mar. 2012.

[7] S. D. Dissanayake and J. Armstrong, "Comparison of ACO-OFDM, DCO-OFDM and ADO-OFDM in IM/DD systems," J. Lightw. Technol., vol. 31, no. 7, pp. 1063-1072, Apr. 1, 2013.

[8] J. Armstrong and A. J. Lowery, "Power efficient optical OFDM," Electron. Lett., vol. 42, no. 6, pp. 370-372, Mar. 2006.

[9] S. C. J. Lee, S. Randel, F. Breyer, and A. M. J. Koonen, "PAM-DMT for intensity-modulated and direct-detection optical communication systems," IEEE Photon. Technol. Lett., vol. 21, no. 23, pp. 1749-1751, Dec. 1, 2009.

[10] D. Tsonev, S. Sinanovic, and H. Haas, "Novel unipolar orthogonal frequency division multiplexing (U-OFDM) for optical wireless," in Proc. IEEE 75th VTC, May 2012, pp. 1-5.

[11] N. Fernando, Y. Hong, and E. Viterbo, "Flip-OFDM for unipolar communication systems," IEEE Trans. Commun., vol. 60, no. 12, pp. 3726-3733, Dec. 2012.

[12] Q. Wang, C. Qian, X. Guo, Z. Wang, D. G. Cunningham, and I. H. White, "Layered ACO-OFDM for intensity-modulated directdetection optical wireless transmission," Opt. Exp., vol. 23, no. 9, pp. 12382-12393, May 2015.

[13] H. Zhang, Y. Yuan, and W. Xu, "PAPR reduction for DCO-OFDM visible light communications via semidefinite relaxation," IEEE Photon. Technol. Lett., vol. 26, no. 17, pp. 1718-1721, Sep. 1, 2014.

[14] N. Huang, J.-B. Wang, J. Wang, C. Pan, H. Wang, and M. Chen, "Receiver design for PAM-DMT in indoor optical wireless links," IEEE Photon. Technol. Lett., vol. 27, no. 2, pp. 161-164, Jan. 15, 2015.

[15] Q. Wang, Z. Wang, and L. Dai, "Iterative receiver for hybrid asymmetrically clipped optical OFDM," J. Lightw. Technol., vol. 32, no. 22, pp. 4471-4477, Nov. 15, 2014. 